\title{
Specific interpretation of augmented feedback changes motor performance and cortical processing
}

\author{
Benedikt Lauber • Martin Keller • Christian Leukel • \\ Albert Gollhofer • Wolfgang Taube
}

\begin{abstract}
It is well established that the presence of external feedback, also termed augmented feedback, can be used to improve performance of a motor task. The present study aimed to elucidate whether differential interpretation of the external feedback signal influences the time to task failure of a sustained submaximal contraction and modulates motor cortical activity. In Experiment 1, subjects had to maintain a submaximal contraction (30\% of maximum force) performed with their thumb and index finger. Half of the tested subjects were always provided with feedback about joint position ( $\mathrm{pF}$-group), whereas the other half of the subjects were always provided with feedback about force (fF-group). Subjects in the $\mathrm{pF}$-group were led to belief in half of their trials that they would receive feedback about the applied force, and subjects in the fF-group to receive feedback about the position. In both groups ( $\mathrm{fF}$ and $\mathrm{pF}$ ), the time to task failure was increased when subjects thought to receive feedback about the force. In Experiment 2, subthreshold transcranial magnetic stimulation was applied over the right motor cortex and revealed an increased motor cortical activity when subjects thought to receive feedback about the joint position. The results showed that the interpretation of feedback influences motor behavior and alters motor cortical activity. The current results support previous studies suggesting a distinct neural control of force and position.
\end{abstract}

B. Lauber $(\bowtie) \cdot$ C. Leukel · A. Gollhofer

Department of Sport Science, University of Freiburg,

Schwarzwaldstr. 175, 79117 Freiburg, Germany

e-mail: benedikt.lauber@sport.uni-freiburg.de

M. Keller · C. Leukel · W. Taube

Department of Medicine, Movement and Sports Science,

University of Fribourg, 1700 Fribourg, Switzerland
Keywords Feedback interpretation - Force control · Position control $\cdot$ Fatigue $\cdot$ Motor cortex

\section{Introduction}

In humans, sensory feedback plays a vital role in controlling movements. The sensory feedback arising from internal sources such as proprioception or vision can be complemented by augmented feedback. The term augmented is used because information is added to the intrinsic feedback that is naturally received by a person's sensory system. It is well established that augmented feedback may enhance performance (Schmidt and Lee 2011). Furthermore, it was shown that performance can be influenced by the content of the provided signal (Lauber et al. 2012). In the latter study, subjects executed a pinching task of thumb and index finger and were provided with two types of visually displayed feedback that were different in their content but otherwise matched as closely as possible (gain, color). In this visuomotor tracking task, a reduced time to task failure and an increased motor cortical (M1) activity were observed when augmented visual feedback was provided about the thumbto-index-finger position compared with feedback about the applied force of the thumb and the index finger (Lauber et al. 2012), whereby the former has been referred to as position feedback $(\mathrm{pF})$ and the latter as force feedback (fF). As the position and the force signal appeared identical when the subjects moved their fingers, we hypothesized that not the content of the signal (force or position) but the interpretation of the external information may be responsible for the differences in the time to task failure. Thus, it could be that as soon as subjects interpreted the provided feedback as force feedback, they used a kind of 'force control mode', whereas the belief to regard position feedback would activate the 
'position control mode'. Thus, the aim of the present study was to test the assumption that interpretation of a visually displayed feedback influences the time to exhaustion when performing a sustained isometric contraction. To test this hypothesis, subjects were provided with an identical feedback, but were led to interpret this feedback in two different ways. The effects of interpretation were tested in two experiments. Experiment 1 assessed the effects of interpretation on performance in a pinching fatiguing task. Experiment 2 assessed the effects of interpretation on the activity of the primary motor cortex using subthreshold TMS.

\section{Methods}

\section{Subjects}

Thirty-two subjects were randomly divided into 3 groups and agreed to participate in two experiments, which were separated by at least $36 \mathrm{~h}$. All subjects were right handed according to the Oldfield Handedness Inventory (Oldfield 1971) and gave their informed consent to the experiment. All experiments were approved by the local ethics committee of the University of Freiburg and were in accordance with the Declaration of Helsinki. None of the subjects had any known neurological or orthopedic disorders.

Seven subjects $(27.1 \pm 0.9$ years, 6 women $)$ participated in the position feedback group (pF-group). The first experiment consisted of the execution of two fatiguing contractions in two sessions, and the second experiment consisted of a subthreshold TMS protocol. This means that the subjects participated in a total of three experimental sessions. The order of the experiments was randomized across subjects.

The first experiment of the fF-group consisted of the execution of two fatiguing contractions in two sessions (eight subjects, $23.3 \pm 0.7$ years; 5 women), and the second experiment consisted of a subthreshold TMS protocol (nine subjects $26.3 \pm 1.0$ years, 4 women). The order of the experiments was randomized across subjects.

The CON-group (eight subjects, $23.6 \pm 0.5$ years; 3 women) was provided with mixed feedback so that subjects received force feedback in half of the trials and position feedback in the other half. Importantly, the CON-group received either position or force feedback without being aware what type of feedback they were actually observing. Subjects in the CON-group were also tested in two experiments: Experiment 1 consisted of two fatiguing contractions conducted in two separate sessions, and Experiment 2 consisted of a subthreshold TMS protocol. This means that the subjects participated in a total of three experimental sessions. This group was included to clarify whether there will be any difference in behavior and in motor cortical activity when no information was given about the source of the augmented feedback.

Setup

Subjects were seated in an upright position in an adjustable chair facing a 21-inch monitor placed $1 \mathrm{~m}$ in front of them. The non-dominant left hand was used in all subjects while the subject's shoulder was abducted so that the forearm could rest on the table placed to their side. The pronated position of the hand and fingers was visually controlled to ensure that the hand of the subjects did not move and that the fingers rested on the table throughout the entire experiment. A custom-built 'hand gripper' was held between thumb and the index finger during the experiment (Fig. 1). The hand gripper had a linear spring-like behavior, that is, the closer the two handles of the gripper were brought, the higher were the produced forces. A force transducer (Tekscan ${ }^{\circledR}$, Inc., South Boston, MA) was rigidly taped to the inside of the thumb during the whole experiment and measured the applied force between thumb and index finger, which was visually presented to the subjects. Additionally, a 2-D angle goniometer (custom-built) was taped to the thumb and index finger to measure the joint angle position. The goniometer was used to present feedback about the joint position between the thumb and the index finger. The hand gripper and the goniometer were so tightly fixed that all changes in finger position resulted in an equal displacement of the hand gripper and the goniometer. The subjects were instructed to press the finger gripper by flexing only the index finger and not the thumb.

After preparation, the subjects performed 3 isometric maximum voluntary contractions (MVC) consisting of a gradual increase in force from zero to maximum over a 3-s time span. The maximal force was held for $2 \mathrm{~s}$, and subjects were verbally encouraged to achieve maximal force. After each trial, there was a rest of $90 \mathrm{~s}$ to allow sufficient time for recovery. The peak force achieved in the 3 trials was considered as the MVC force value. Subsequently, $30 \%$ of the MVC value was calculated and represented as red target line on the computer screen, and subjects were asked to meet this line with a black line corresponding to the actual exerted force or the actual position while pressing the hand gripper. The target line for the $\mathrm{pF}$-group corresponded to the individual thumb-to-index angle when the subjects matched the force level of $30 \%$ MVC (Fig. 1). All subjects were instructed to sustain the contractions until task failure. Task failure was determined when subjects were not able to hold the force within $5 \%$ of the target force over a period of $5 \mathrm{~s}$ (fF-group) or when they were not able to keep the thumbto-index-finger angle within $5 \%$ of the target angle for $5 \mathrm{~s}$ (pF-group) despite strong verbal encouragement (in line with Klass et al. 2008; Rudroff et al. 2010). 


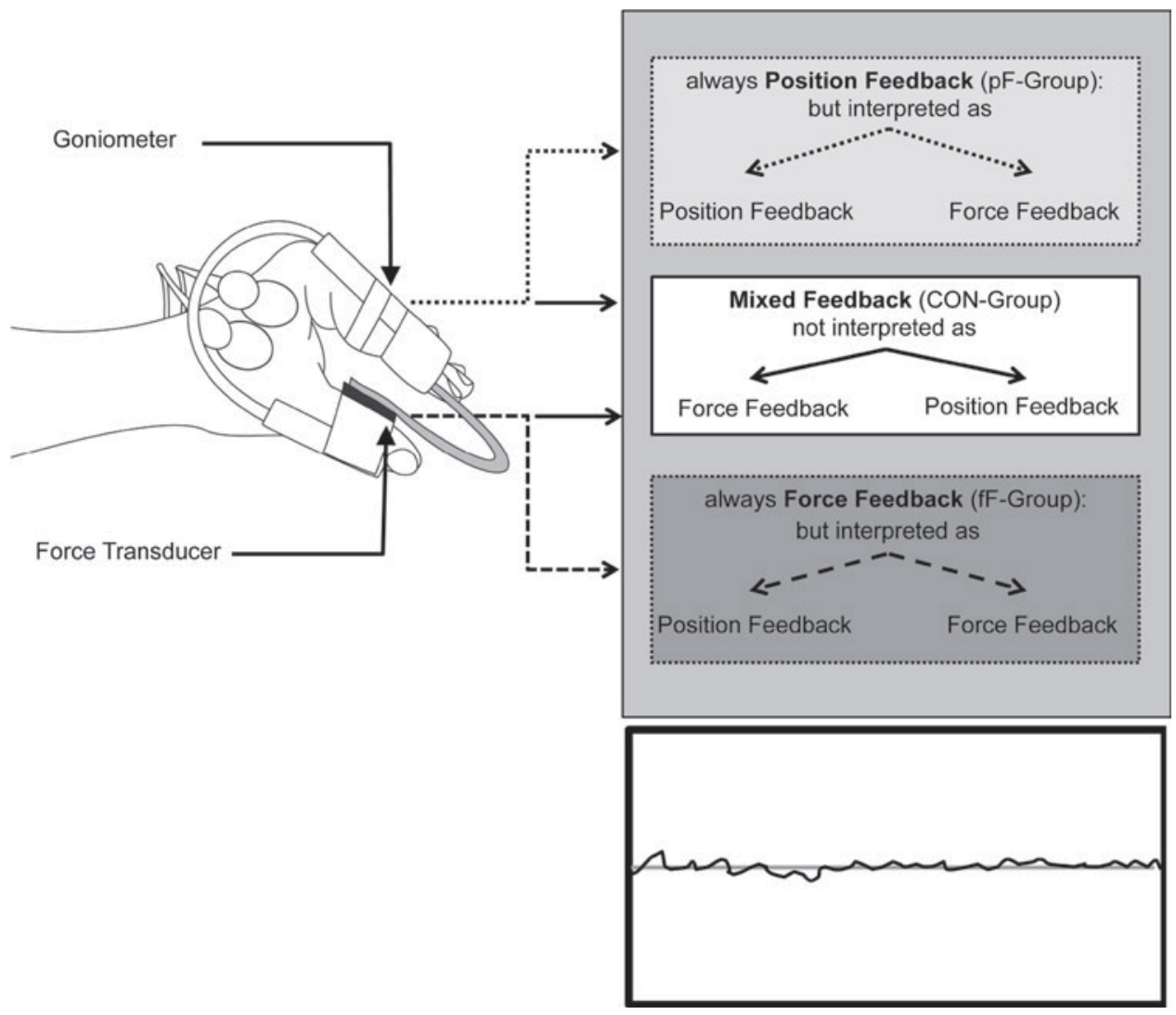

Fig. 1 Schematic drawing of the experimental setup including the groups. The hand gripper was held between thumb and index finger while a force transducer was taped to the inside of the thumb and a goniometer was taped on the thumb and index finger (from Lauber et al. 2012). The pF-group always received feedback about joint position, and subjects were led to believe they received force feedback in $50 \%$ of their trials. The fF-group always received feedback about the applied force, and subjects were led to believe

\section{Feedback presentation}

During the contractions, either the force or the position signal was visually displayed on a computer screen placed in front of the subjects. The position feedback and the force feedback could not be differentiated from each other as they were displayed in the same way (line thickness $0.3 \mathrm{~cm}$; a force of $1 \mathrm{~N}$ caused a $1.25-\mathrm{mm}$ movement of the hand gripper corresponding to 0.6 degrees), and this relation was linear over the applied range of force and position, respectively. The scale of the two types of feedback was adjusted so that movements between thumb and index finger resulted in the same deviation of the force and the position signal from the baseline. The signal-to-noise ratio was high, and the variability of the signal was low so that we could unambiguously clarify the time of exhaustion of the subjects and that the subjects had no problems to maintain force/position in Experiment 2. Subjects were verbally instructed to match that they received position feedback in half of their trials. The CON-group received force and position feedback, which was alternatively mixed (CON-group), but the subjects were not aware about changes in the feedback. The box at the bottom is an illustration of how the feedback was presented. The gray line represents the target line, and the black line represents the actual curve (force/position) produced by the subjects when pressing the hand gripper

the target line that was presented on the screen with the line they could modify by pressing the hand gripper. Furthermore, subjects had to keep either the force or the position as constant as possible by matching the line produced by pressing the hand gripper with the target line. The setup did not allow subjects to detect from where the feedback was coming, that is, from the force transducer or the goniometer.

The force and position signals were stored on a PC with a sampling frequency of $4 \mathrm{kHz}$, and the same setup has previously been used (Lauber et al. 2012).

\section{Procedures}

\section{EMG recording}

After preparation of the skin, bipolar surface electrodes (Blue sensor P, Ambu ${ }^{\circledR}$, Bad Nauheim, Germany) were attached to the skin over the first dorsal interosseus muscle 
(FDI) and the abductor pollicis brevis muscle (APB) of the left hand with $2-\mathrm{cm}$ interelectrode distance. The reference electrode was placed on the olecranon of the same arm. The EMG recordings were amplified $(1,000 \times)$, bandpass-filtered (10-1 kHz), and sampled at $4 \mathrm{kHz}$. All data were stored on computer using custom-built software (LabView based, National Instruments, Austin, TX) for offline analysis.

\section{Experiment 1: Sustained contractions}

Experiment 1 aimed to elucidate interpretation-specific differences in the time to task failure of submaximal sustained contractions. Subjects from the pF-group and the fF-group participated in two fatiguing contractions divided into two separate experimental sessions. In one session, they were told to receive feedback about the applied force ( $\mathrm{pF}$-group), whereas in the other session, they were told to receive feedback about the position (fF-group). In fact, subjects exclusively received either feedback about their position (pF-group) or feedback about the applied force (fF-group). Thus, in one of the sustained contractions, subjects received correct feedback, whereas in the second session, they received incorrect feedback (tricked feedback) without being aware of it. The $\mathrm{CON}$-group received during one sustained contraction force feedback and during the other position feedback without verbal instructions regarding the type/ nature of the feedback. The order of the sessions, that is, whether trials started with force or position feedback, was randomly altered in all groups.

\section{Experiment 2: Subthreshold TMS}

Experiment 2 of the present study aimed to reveal differences in motor cortical activity with respect to the interpretation of the type of feedback. For this purpose, subthreshold TMS of the right motor cortex (M1) was used. Similar to Experiment 1 , subjects from the $\mathrm{pF}$-group exclusively received feedback about their joint position (Fig. 1) and subjects from the fF-group solely received feedback about the applied force (Fig. 1). However, subjects were again told to receive force feedback in half of the trials and position feedback in the other half. Thus, they were led to believe to receive different feedback modalities, while in reality they performed identical tasks with identical feedback.

In order to clarify whether the interpretation of the feedback and/or the feedback source (force or position) caused the alterations in M1 activity, the CON-group received random feedback, that is, subjects received force feedback in half of the trials and position feedback in the other without specific information about the feedback source and without instruction on how to perform the contractions, that is, to control contractions on a force or position basis.
The experimental setup was the same as in Experiment 1 and followed the same procedure to determine the target values (i.e., $30 \%$ of MVC). The main difference in this protocol was that the duration of the contractions was short and therefore not exhaustive (approximately $80 \mathrm{~s}$ for one trial). In the course of the 80-s trials, 40 sweeps with TMS and 40 sweeps without TMS were applied resulting in a TMSevoked response in half of the data pieces, whereas in the other half no stimulation was applied in order to determine the background EMG activity. The interstimulus interval ranged from 0.8 to $1.1 \mathrm{~s}$ and was similar to the intervals used in previous experiments (Zuur et al. 2010; Lauber et al. 2012). To avoid fatigue, a break of 3 min was given between the trials. To analyze whether TMS caused a facilitation or suppression in the FDI EMG, the rectified and then averaged 40 sweeps without stimulation (control EMG) were subtracted from 40 sweeps with stimulation (Davey et al. 1994; Petersen et al. 2001; Zuur et al. 2010; Lauber et al. 2012). The intensity of the TMS during the first trial was chosen to be high enough to evoke MEPs in the FDI (0.9 times motor threshold at rest). After this block, subjects were asked to relax and the stimulation intensity was then gradually decreased before the next block started after a pause of $3 \mathrm{~min}$. In this manner, the stimulus intensity was further decreased until a suppression of the EMG was visible without the presence of any facilitation (Petersen et al. 2001; Zuur et al. 2010; Lauber et al. 2012). This adjustment served to reveal the maximal suppression by subthreshold TMS in all conditions. Finally, the stimulus intensity was further decreased until no difference between the averaged sweeps with and without stimulation could be observed (i.e., no effect of TMS), resulting in an average of $5.5 \pm 1$ trials for each feedback condition.

\section{Data analyses}

For all protocols, maximal EMG activity (EMGmax) was calculated as the root-mean-square value taken over a 0.5 -s interval around the peak force achieved during the MVC test. The EMGmax was assessed during the same experimental session with the identical setup in advance of the fatiguing contractions (fF- and pF-groups) and the subthreshold TMS measurements (fF-, pF-, and CON-groups), respectively.

\section{Experiment 1}

During the sustained contractions, muscular activity was quantified by root-mean-square values of the rectified EMG over $8 \mathrm{~s}$ measured every $30 \mathrm{~s}$ during the course of the sustained contractions and normalized to EMGmax. To compare changes in EMG activity, the first $8 \mathrm{~s}$ of the sustained contraction was compared with the last $8 \mathrm{~s}$. Changes in coactivation of muscles of the initial $8 \mathrm{~s}$ [EMG (\%EMGmax 
for FDI/APB) and the final $8 \mathrm{~s}$ of the sustained contraction were calculated. Furthermore, the increase in FDI EMG activity over time was quantified as percentage of the initial value (first $\mathrm{min}$ ) and was calculated for every minute of the contraction. Additionally, the force signals were analyzed in order to evaluate the steadiness of the sustained contraction. For this purpose, the force signal, which was measured with the same force transducer in the fF- and the $\mathrm{pF}$-groups, was quantified as the coefficient of variation for the first 3 min of each sustained contraction.

\section{Experiment 2}

For the data of the subthreshold TMS protocol, the onset of the EMG suppression caused by subthreshold TMS was defined as the instant where the averaged EMG for the stimulated condition was less than the control EMG for at least $4 \mathrm{~ms}$ in a time window of 20 to $50 \mathrm{~ms}$ after the application of the TMS stimulus. The end of the suppression was defined as the instant when the stimulated EMG was above the control EMG for more than $1 \mathrm{~ms}$. The mean suppression was expressed as percentage change (control- stimulated)/mean control $^{*} 100$ ). The control EMG was averaged in the same time window that was defined for the subthreshold TMS analyses. This method of quantifying suppression has been used in recent papers using the same technique (Zuur et al. 2010; Lauber et al. 2012). Peak-to-peak amplitude of the MEP was measured in a window of $50 \mathrm{~ms}$ after the application of the TMS stimulus.

\section{Statistical analyses}

Before comparing the variables, normal distribution of the data was tested using the Shapiro-Wilk test $(p=0.29)$. All data are reported as mean \pm standard error of the mean (SEM). For Experiment 1, a 3-way repeated-measures ANOVA was performed to assess differences in the time to task failure. Thus, the ANOVA was calculated with the within-subject factor of the SOURCE OF INFORMATION (force transducer/goniometer) and the between-subject factors INTERPRETATION (force/position) to identify differences in interpretation-specific time to task failure of the sustained contraction, and GROUP (pF, fF, and CON). For the EMG, repeated-measures ANOVA with factors MUSCLE $($ FDI/APB) $\times$ INTERPRETATION (force/position) $\times$ TIME (initial $8 \mathrm{~s}$ vs. last $8 \mathrm{~s}$ ) was conducted.

Post-hoc corrected (Bonferroni) paired Student's $t$ tests were used to test within-group effects of the interpretation of feedback on time to task failure (force/position) and EMG activity (force/position). EMGmax, coefficient of variation, and MVC values were compared using Bonferronicorrected two-sided paired $t$ tests.
For Experiment 2, interpretation-specific differences in EMG suppression were calculated using repeated-measures ANOVA with factors INTERPRETATION (force/position) and GROUP (pf, fF, and CON). Post-hoc Bonferronicorrected paired Student's $t$ tests were used to test withingroup effects of the interpretation of feedback within the fF- pF-, and CON-groups. SPSS 19.0 software was used for all statistical analyses $\left(\right.$ SPSS $^{\circledR}$, Chicago, IL), and the level of significance was set at $p \leq 0.05$.

\section{Results}

Experiment 1

When subjects interpreted the feedback as force feedback, the time to task failure was significantly prolonged (INTERPRETATION main effect; $F_{1,17}=12.2 ; \eta^{2}=0.41$; $p<0.01)$. Furthermore, a significant group effect between the $\mathrm{fF}$ - and $\mathrm{pF}$-groups and the $\mathrm{CON}$-group was apparent as the CON-group showed no differences in the time to task failure (GROUP main effect; $F_{1,17}=4.4 ; \eta^{2}=0.34$; $p<0.01)$.

\section{$p F$-group}

Although the test conditions of the tricked and correct feedback trials were identical, subjects displayed significantly longer times to task failure when they were told that they controlled force instead of position (correct feedback $7.8 \pm 1.4 \mathrm{~min}$ vs. tricked feedback $14.6 \pm 2.7 \mathrm{~min}$; $p=0.05$; Fig. 2).

FDI EMG activity significantly increased in the course of the sustained contractions (TIME main effect; $F_{1,12}=20.7$; $\left.\eta^{2}=0.63 ; p=0.01\right)$, but was not interpretation specific $\left(\right.$ INTERPRETATION $\times$ TIME; $F_{1,12}=0.01 ; \eta^{2}=0.01$; $p=0.9)$. FDI EMG increased by $20.4 \pm 5.5 \%$ in the correct feedback condition $(p=0.01)$ and by $21.5 \pm 7.4 \%$ ( $p=0.02$; Table 1$)$ in the tricked feedback condition.

However, the rate of EMG increase over time was significantly greater when subjects interpreted the feedback as position feedback (correct feedback $6.1 \pm 2.3 \% / \mathrm{min}$ vs. tricked feedback $2.0 \pm 0.7 \% / \mathrm{min} ; p=0.05)$. The EMG data show that the increase in EMG activity at the time of task failure was comparable in force- and position-controlled trials, but that the increase and the time to task failure occurred much faster as soon as subjects thought they should control position.

The APB EMG activity increased in the course of the sustained contractions (TIME main effect; $F_{1,12}=9.1$; $\left.\eta^{2}=0.43 ; p=0.01\right)$, but was not interpretation specific $\left(\right.$ INTERPRETATION $\times$ TIME; $F_{1,12}=1.0 ; \eta^{2}=0.83$; $p=0.31)$. The APB EMG showed a trend toward an increase 

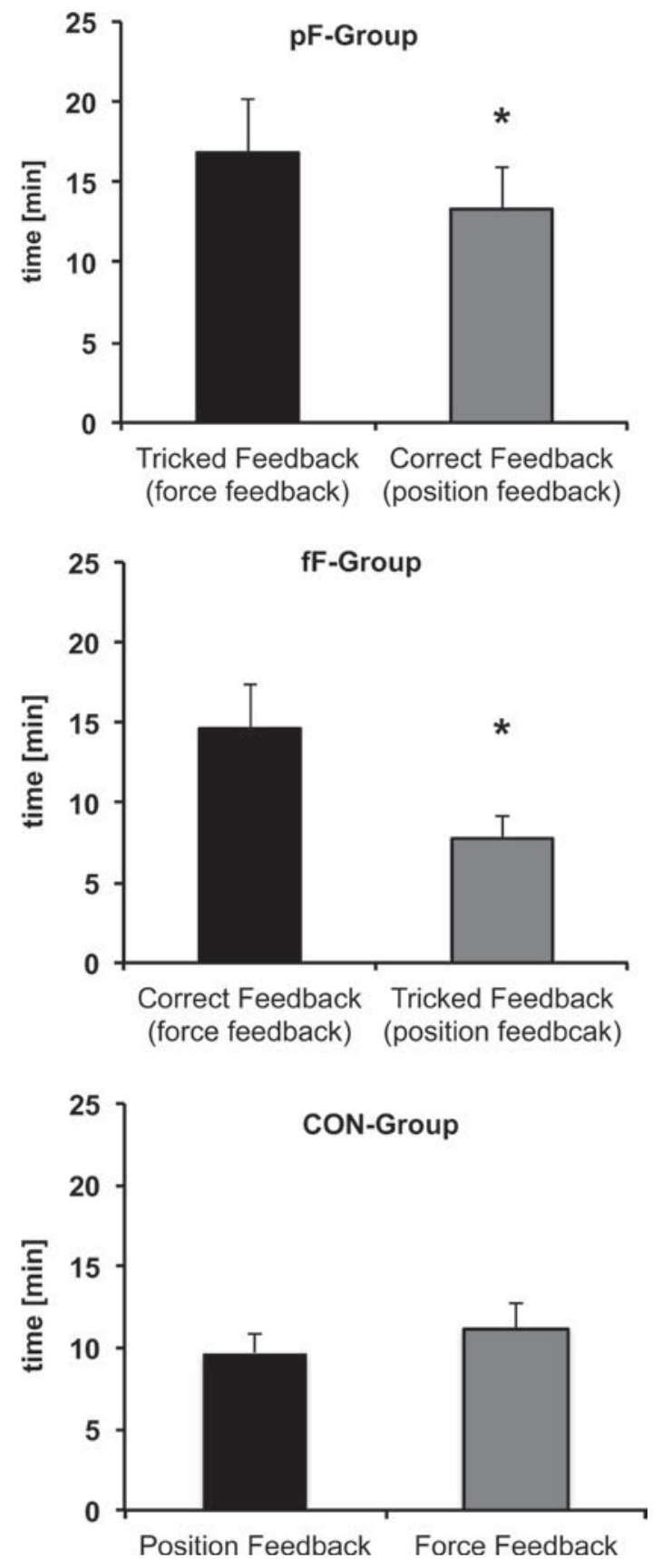

Fig. 2 Time to task failure of the sustained contractions. As soon as subjects from the pf-group interpreted the feedback as force feedback, the time to task failure was significantly longer compared with when they interpreted it as position feedback $(* p=0.02)$. This was also the case for the fF-group, showing an increased time to task failure when they interpreted the feedback as force feedback compared with when they interpreted the feedback as position feedback $\left({ }^{*} p=0.05\right)$. The CON-group showed no differences between the contractions

by $16.2 \pm 7.1 \%(p=0.06)$ in the correct feedback condition and by $7.0 \pm 3.7 \%(p=0.07)$ in the tricked feedback condition (Table 1).
The rate of EMG activity over time was not different between the conditions (correct feedback $2.2 \pm 0.8 \% / \mathrm{min}$ vs. tricked feedback $1.2 \pm 0.5 \% / \mathrm{min} p=0.48$ ).

There was no difference in the initial EMGmax values of the FDI (tricked feedback $0.56 \pm 0.1 \mathrm{mV}$ vs. correct feedback $0.56 \pm 0.2 \mathrm{mV} ; p=0.99$ ) and the APB (tricked feedback $0.48 \pm 0.4 \mathrm{mV}$ vs. correct feedback $0.30 \pm 0.11 \mathrm{mV}$; $p=0.18$ ) obtained during initial MVC. The pF-group displayed no differences in muscle co-activation between the tricked feedback-controlled and the correct feedbackcontrolled contractions (start $p=0.60$; end: $p=0.70$ ). There were also no differences in MVC values (force $34.5 \pm 13.0 \mathrm{~N}$ vs. position $33.1 \pm 12.5 \mathrm{~N} ; p=0.37$ ). The coefficient of variation in the force signal revealed no difference between the sustained contractions (tricked vs. correct feedback; $p=0.95$ ).

fF-group

In this group, subjects always received feedback about their force. Again, subjects displayed significantly longer times to task failure when they were told that they controlled force instead of position (correct feedback $16.8 \pm 3.4 \mathrm{~min}$ vs. tricked feedback $13.3 \pm 2.6 \mathrm{~min} ; p=0.02$; Fig. 2).

FDI EMG activity did significantly increase in the course of the sustained contractions (TIME main effect; $\left.F_{1,14}=30.30 ; \eta^{2}=0.68 ; p=0.001\right)$, but was not interpretation specific (INTERPRETATION $\times$ TIME; $\left.F_{1,14}=2.3 ; \eta^{2}=0.16 ; p=0.14\right)$. At the time of task failure, FDI EMG was increased by $18.1 \pm 5.0 \%$ in the correct feedback condition $(p<0.01)$ and by $32.2 \pm 7.6 \%$ $(p<0.01)$ in the tricked feedback condition (Table 1).

The rate of EMG increase over time, however, was significantly greater when subjects interpreted the feedback as position feedback (correct feedback $1.2 \pm 0.5 \% / \mathrm{min}$ vs. tricked feedback $3.5 \pm 1.3 \% / \mathrm{min} ; p=0.05$ ). Thus, the initial observation made in the $\mathrm{pF}$-group was confirmed in the fF-group: the increase in EMG activity at the time of task failure was comparable in force- and position-controlled trials, but the increase in EMG activity and the time to task failure occurred much faster as soon as subjects thought they should control the position.

The APB EMG activity increased in the course of the sustained contractions (TIME main effect; $\left.F_{1,14}=19.7 ; \eta^{2}=0.58 ; p=0.01\right)$, but was not interpretation specific (INTERPRETATION x TIME; $F_{1,14}=0.01$; $\left.\eta^{2}=0.001 ; p=0.85\right)$. APB EMG increased by $18.8 \pm 6.0 \%(p=0.01)$ in the correct feedback condition and by $20.0 \pm 6.3 \%(p=0.01)$ in the tricked feedback condition (Table 1). The rate of EMG activity over time was not different between the conditions (correct feedback $1.2 \pm 0.5 \% / \mathrm{min}$ vs. tricked feedback $2.3 \pm 0.8 \% / \mathrm{min}$; $p=0.47)$. 
Table 1 EMG of the sustained contractions

\begin{tabular}{|c|c|c|c|c|c|c|}
\hline & \multicolumn{2}{|l|}{ pF-Group $(n=7)$} & \multicolumn{2}{|l|}{ fF-Group $(n=8)$} & \multicolumn{2}{|l|}{ CON-Group $(n=5)$} \\
\hline & Force & Position & Force & Position & Force & Position \\
\hline $\begin{array}{l}\text { FDI EMG } \\
\text { [\% EMGmax] } \\
\text { (start vs. end) }\end{array}$ & $\begin{array}{c}28.1 \pm 4.9 \text { versus } \\
49.6+9.1^{*}\end{array}$ & $\begin{array}{c}26.9 \pm 3.9 \text { versus } \\
47.4+9.2^{*}\end{array}$ & $\begin{array}{c}27.6 \pm 3.9 \text { versus } \\
46.0 \pm 6.0^{*}\end{array}$ & $\begin{array}{c}21.3 \pm 4.2 \text { versus } \\
53.4+8.8^{*}\end{array}$ & $\begin{array}{c}18.5 \pm 7.1 \text { versus } \\
53.6 \pm 8.2^{*}\end{array}$ & $\begin{array}{c}16.1 \pm 4.2 \text { versus } \\
43.7 \pm 6.3^{*}\end{array}$ \\
\hline $\begin{array}{l}\text { APB EMG } \\
\text { [\% EMGmax] } \\
\text { (start vs. end) }\end{array}$ & $\begin{array}{l}19.4 \pm 6.6 \text { versus } \\
23.5+7.7\end{array}$ & $\begin{array}{l}24.1 \pm 6.1 \text { versus } \\
32.2 \pm 6.4\end{array}$ & $\begin{array}{c}19.5 \pm 2.6 \text { versus } \\
38.3 \pm 6.6^{*}\end{array}$ & $\begin{array}{c}21.4 \pm 4.7 \text { versus } \\
41.3 \pm 8.5^{*}\end{array}$ & $\begin{array}{l}41.6 \pm 14.5 \text { versus } \\
52.8 \pm 11.2^{\#}\end{array}$ & $\begin{array}{c}17.7 \pm 3.7 \text { versus } \\
29.5 \pm 3.6^{* *}\end{array}$ \\
\hline
\end{tabular}

FDI EMG activity increased in the course of the fatiguing contraction in the pF-group when subjects interpreted the feedback as force feedback $(* p=0.02)$ and when they interpreted the feedback as position feedback $(* p=0.01)$. This was also true in the fF-group (force $* p=0.01$; position $* p=0.01$ ) and the CON-group (force $* p=0.002$; position $* p=0.01$ ). APB EMG activity also significantly increased in the $\mathrm{pF}$ group for both conditions (force $* p<0.05$; position $* p<0.05$ ), whereas there was only a trend toward an increase in the $\mathrm{pF}$-group (force $p=0.06$; position $p=0.07)$. In the CON-group, EMG significantly increased during the position-controlled contraction $(* * p=0.001)$ and had a trend in the force-controlled contraction $\left({ }^{\#} p=0.08\right)$

There was also no difference in the initial EMGmax values of the FDI (correct feedback $0.61 \pm 0.2 \mathrm{mV}$ vs. tricked feedback $0.54 \pm 0.2 \mathrm{mV} ; p=0.14)$ and the APB (correct feedback $0.50 \pm 0.2 \mathrm{mV}$ vs. tricked feedback $0.36 \pm 0.1 \mathrm{mV} ; p=0.21$ ) obtained during MVC.

Furthermore, there was no difference in muscle co-activation between the tricked feedback-controlled and the correct feedback-controlled contractions (start $p=0.64$; end $p=0.73)$. There were also no differences in MVC values (force $51.0 \pm 18.0 \mathrm{~N}$ vs. position $51.1 \pm 18.4 \mathrm{~N} ; p=0.32$ ). The coefficient of variation in the force signal revealed no difference between the sustained contractions (tricked vs. correct feedback, $p=0.21$ ).

\section{CON-group}

Subjects in this group displayed no difference in time to task failure (position feedback $11.1 \pm 1.6 \mathrm{~min}$ vs. force feedback $9.7 \pm 1.2 \mathrm{~min} ; p=0.58$; Fig. 2 ).

FDI EMG activity did significantly increase in the course of the sustained contractions (TIME main effect; $F_{1,8}=56.7$; $\eta^{2}=0.87 ; p<0.01$ ), but was not feedback specific (INTERPRETATION x TIME; $F_{1,12}=0.80 ; \eta^{2}=0.09 p=0.80$ ). FDI EMG increased by $20.7 \pm 7.5 \%$ in the position feedback condition $(p=0.01)$ and by $35.1 \pm 5.5 \%(p<0.01)$ in the force feedback condition (Table 1).

The rate of EMG increase over time was also comparable between the tasks (position feedback $3.2 \pm 2.3 \% / \mathrm{min}$ vs. force feedback $4.9 \pm 2.2 \% / \mathrm{min} ; p=0.34$ ).

The APB EMG activity also increased in the course of the sustained contractions (TIME; $F_{1,8}=21.1$; $\eta^{2}=0.72 ; p<0.01$ ), but was not feedback specific (INTERPRETATION $\times$ TIME; $F_{1.8}=0.03 ; p=0.85$ ). APB EMG increased by $12.2 \pm 1.5 \%(p<0.01)$ in the position feedback condition, and there was a trend toward an increase by $11.1 \pm 4.8 \%(p=0.08)$ in the force feedback condition (Table 1).
The rate of EMG activity over time was not different between the conditions (position feedback $1.8 \pm 0.8 \% / \mathrm{min}$ vs. force feedback $1.5 \pm 0.7 \% / \mathrm{min} ; p=0.68$ ).

There was no difference in the initial EMGmax values of the FDI (position feedback $0.53 \pm 0.2 \mathrm{mV}$ vs. force feedback $0.60 \pm 0.2 \mathrm{mV} ; p=0.47$ ) and the APB (position feedback $0.34 \pm 0.1 \mathrm{mV}$ vs. force feedback $0.19 \pm 0.1 \mathrm{mV} ; p=0.17$ ) obtained during initial MVC. The CON-group displayed no differences in muscle co-activation between the position feedback-controlled and the force feedback-controlled contractions ( $\operatorname{start} p=0.1$; end $p=0.6$ ). There were also no differences in MVC values (force $38.0 \pm 16.9 \mathrm{~N}$ vs. position $37.6 \pm 16.8 \mathrm{~N} ; p=0.75)$. The coefficient of variation in the force signal revealed no difference between the sustained contractions (position feedback vs. force feedback; $p=0.31$.

\section{Experiment 2}

When subjects interpreted the feedback as position feedback, the ANOVA revealed a significant INTERPRETATION effect of the data obtained with subthreshold TMS (INTERPRETATION main effect; $F_{1,21}=24.0 ; \eta^{2}=0.53$; $p<0.001)$. Furthermore, a significant group effect was apparent as the CON-group showed no differences in the EMG suppression (GROUP main effect; $F_{2,21}=10.0$; $\left.\eta^{2}=0.48 ; p=0.01\right)$.

\section{$p F$-group}

In all seven subjects who participated in this experiment, the use of subthreshold TMS onto the motor cortex resulted in a significant suppression of the FDI EMG in both parts of the experiment. Table 2 shows the mean, onset, and duration of the suppression as well as the onset of the MEP and the background EMG activity. The mean suppression was significantly greater when subjects were told to control 
Table 2 Results obtained using subthreshold TMS

\begin{tabular}{|c|c|c|c|c|c|c|}
\hline & \multicolumn{2}{|c|}{ pF-Group $(n=7)$} & \multicolumn{2}{|c|}{ fF-Group $(n=8)$} & \multicolumn{2}{|c|}{ CON-Group $(n=8)$} \\
\hline & Force & Position & Force & Position & Force & Position \\
\hline $\begin{array}{l}\text { Mean suppression } \\
\text { [\% control EMG] }\end{array}$ & $31.69 \pm 3.81$ & $36.67 \pm 3.19^{*}$ & $22.99 \pm 2.77$ & $31.66 \pm 2.28 * * *$ & $22.01 \pm 2.76$ & $21.35 \pm 2.55$ \\
\hline $\begin{array}{l}\text { Onset suppression } \\
{[\mathrm{ms}]}\end{array}$ & $37.86 \pm 2.68$ & $38.21 \pm 2.52$ & $33.33 \pm 2.78$ & $33.69 \pm 3.27$ & $29.44 \pm 1.44$ & $30.51 \pm 1.86$ \\
\hline $\begin{array}{l}\text { Duration suppression } \\
\text { [ms] }\end{array}$ & $15.57 \pm 1.37$ & $16.39 \pm 1.36$ & $17.00 \pm 2.13$ & $19.53 \pm 2.27$ & $9.41 \pm 1.60$ & $8.53 \pm 1.52$ \\
\hline Onset MEP [ms] & $23.00 \pm 0.49$ & $22.04 \pm 0.48$ & $21.97 \pm 0.78$ & $22.39 \pm 0.90$ & $21.69 \pm 1.29$ & $22.25 \pm 1.00$ \\
\hline bEMG [mv] & $0.14 \pm 0.01$ & $0.14 \pm 0.01$ & $0.06 \pm 0.01$ & $0.07 \pm 0.01$ & $0.09 \pm 0.01$ & $0.08 \pm 0.01$ \\
\hline
\end{tabular}

The subthreshold TMS resulted in an EMG suppression in all three feedback protocols. In the pF-group, the mean EMG suppression was significantly greater when subjects interpreted the feedback as position feedback $(* p=0.02)$ and not as force feedback. This was also the case for the fF-group ( $\left.{ }^{* * *} p=0.001\right)$, whereas there was no difference in the CON-group $(p=0.58)$. The onset and the duration of the suppression were not different within the protocols, which was also true for the onset of the MEP and the background EMG activity

position compared with the force-controlled contraction ( $p=0.02$; Fig. 3). There was no difference in the onset of the suppression $(p=0.66)$, the duration of the suppression $(p=0.26)$, the onset of the MEP $(p=0.15)$ as well as the background EMG $(p=0.97)$.

\section{fF-group}

In all nine subjects who participated in this experiment, the use of subthreshold TMS onto the motor cortex resulted in a significant suppression of the FDI EMG for the correct and the tricked feedback conditions. Table 2 shows the mean, onset, and duration of the suppression as well as the onset of the MEP and the background EMG activity. Again, mean suppression was significantly greater when subjects were led to believe to control position instead of force $(p<0.01$; Fig. 3). There was no difference in the onset of the suppression $(p=0.75)$, the duration of the suppression $(p=0.25)$, the onset of the MEP $(p=0.38)$ as well in the background EMG $(p=0.65)$.

\section{CON-group}

In all eight subjects who participated in this experiment, the use of subthreshold TMS onto the motor cortex resulted in a significant suppression of the FDI EMG during the positioncontrolled and the force-controlled tasks. Table 2 shows the mean, onset, and duration of the suppression as well as the onset of the MEP and the background EMG activity. There was no difference in mean suppression between the position-controlled and the force-controlled contractions $(p=0.58$; Fig. 3). Furthermore, there was no difference in the onset of the suppression $(p=0.47)$, the duration of the suppression $(p=0.09)$, the onset of the MEP $(p=0.15)$, and the background EMG $(p=0.19)$.

\section{Discussion}

The present study aimed to elucidate whether the interpretation of feedback influences the time to task failure of sustained submaximal contractions (Experiment 1) and modulates motor cortical activity (Experiment 2).

The results from Experiment 1 showed a prolonged time to task failure when subjects interpreted the feedback as force feedback compared with when they interpreted it as position feedback. This effect occurred likewise whether subjects always received force feedback (fF-group) or position feedback (pF-group).

Experiment 2 revealed that the cortical activity was altered by the interpretation of feedback as the EMG suppression, which was induced by subthreshold TMS, was greater when subjects interpreted the feedback as position feedback. Again, this finding can be attributed to the interpretation of the external information and was independent from the source of the feedback as it was similarly evident in the $\mathrm{fF}$ - and $\mathrm{pF}$-groups.

The current experiments highlight for the first time that force and position control strategies can be 'separately activated' for one and the same task. Furthermore, the interpretation of the source of the feedback seems to determine which control strategy is used as the control group receiving no information regarding the source of the feedback showed no feedback-specific differences in motor behavior and M1 activity.

Interpretation of feedback influences motor behavior

It is not new that the provision of augmented feedback can influence motor performance. For instance, subjects instantly augmented their force capacity when provided with augmented feedback $(\mathrm{aF})$ about their actual force/torque 
EMG

Suppression
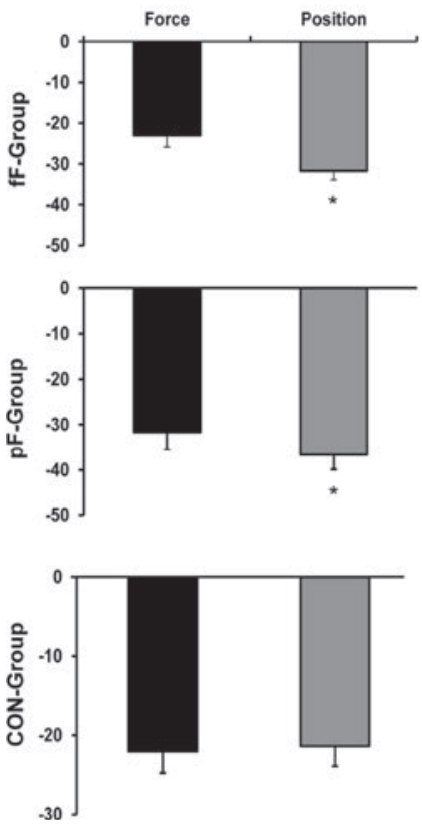
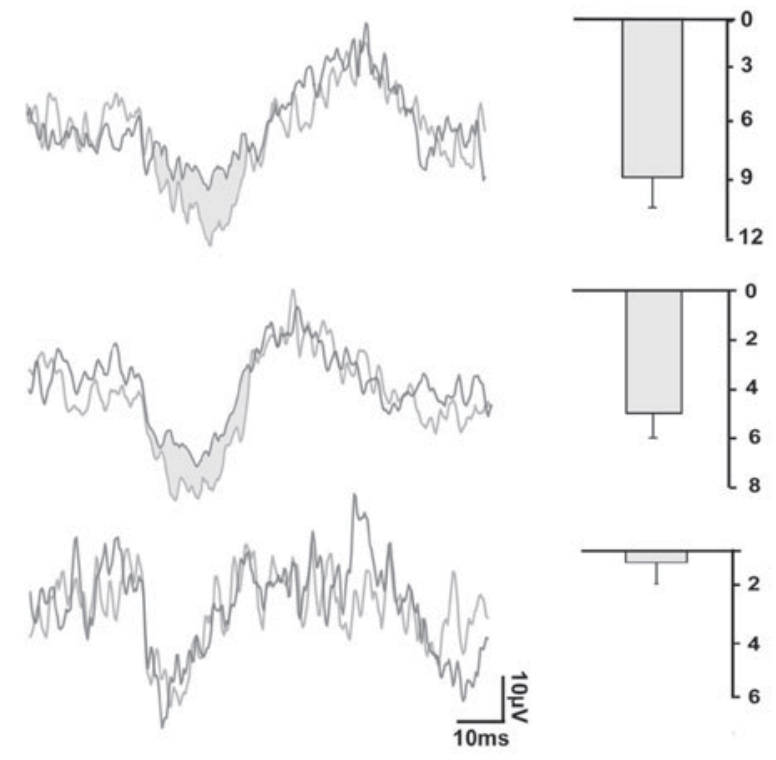

Difference in EMG suppresion [\%]

Fig. 3 Group mean date of EMG suppression. Left Mean values of the EMG suppression caused by subthreshold TMS. EMG (as \% of control EMG) suppression was significantly greater when subjects of the fF-group interpreted the feedback as position feedback compared with when they interpreted the feedback as force feedback $(* * * p=0.001)$. For the pF-group, EMG suppression was significantly greater when subjects interpreted the feedback as position feedback compared with when they interpreted the feedback as force feedback $(* p=0.02)$. There was no difference in the EMG suppression for the CON-group when they received force feedback and position feedback $(p=0.58)$. Middle Mean FDI EMG per group.

compared with when no feedback was presented (Peacock et al. 1981; Hopper 2003). Furthermore, aF was also shown to influence the training outcome when applied during several weeks of tennis training (Moran et al. 2011) and during rehabilitation (Langhorne et al. 2009). Thus, augmented feedback constitutes a powerful tool to improve motor performance and motor learning. So far, most of the previous studies focused on feedback-related aspects such as the type of feedback, the timing of feedback, or the frequency of feedback (for an overview, please see Schmidt and Lee 2011). In contrast, the current study focused on a new aspect of feedback, namely how the instruction about the informational content of the signal, and consequently the interpretation of it, influences motor behavior. Our data show that not only the presence and/or the content of augmented feedback but also its interpretation influenced task performance. As the mechanical setup, the presentation (color, gain) and information content of the feedback (force or position signal) were kept identical within the groups ( $\mathrm{pF}$ and $\mathrm{fF}$, respectively), these factors
Black and grey lines indicate how subjects interpreted the feedback. The gray area highlights the difference in EMG suppression. Right Difference in EMG suppression between the correct feedback and the tricked feedback. When subjects of the fF-group interpreted the feedback as position feedback, EMG suppression was greater $(8.09 \pm 1.89 \%)$ compared with when they interpreted the feedback as force feedback. Similarly, when subjects of the pF-group interpreted the feedback as position feedback, EMG suppression was also greater $(4.98 \pm 1.65 \%)$ compared with when they interpreted the feedback as force feedback

cannot be responsible for the differences between the force- and position-controlled contractions. Thus, it seems most likely that subjects used a 'force control mode' and a 'position control mode' dependent on the information they verbally received about the feedback. In the 'position control mode', the time to task failure was reduced, but not in the 'force control mode,' irrespective of the actual feedback.

Interpretation of feedback influences neural control

In the current study, we furthermore hypothesized that a different interpretation of the same feedback would change the neural activity within M1. The motor cortex seems to be a worthwhile target as it is directly involved in the control of voluntary, goal-directed movements (Porter and Lemon 1993; Scott 2003) like the task executed in the present study.

This hypothesis was confirmed by the electrophysiological results of Experiment 2 (subthreshold TMS), supporting 
the notion that neurons in M1 are differently activated in force- and position-controlled tasks. The differential control in force and position tasks was evidenced by interpretation specific suppression in response to subthreshold TMS. The general idea underlying TMS at stimulation intensities below the threshold to evoke a motor response (i.e., MEP) is to activate low-threshold intracortical inhibitory interneurons which then act via their synaptic input on the excitability of corticospinal cells reducing the cortical drive present during voluntary movements (Di Lazzaro et al. 1998; Petersen et al. 2010). This can then be seen as a reduction in the ongoing EMG activity. The notion that this is a strictly cortical effect is supported by findings reporting the absence of any descending volleys measured with high cervical epidural electrodes in conscious humans (Di Lazzaro et al. 1998).

The greater suppression in the position-controlled contractions in the present study indicates that the susceptibility of intracortical inhibitory interneurons or corticomotoneurons was augmented as soon as subjects used the 'position control mode.' Previous studies have further argued that greater suppression caused by sub-TMS indicates greater motor cortical involvement (Seifert and Petersen 2010). In line with this assumption, greater motor cortical activity in the present experiment may also explain the faster increase in EMG activity in the position-controlled contractions. The faster increase in EMG activity indicates a faster recruitment of motor units, which may be realized by a stronger motor cortical activity (Löscher and Nordlund 2002). Such a stronger motor cortical activity would also explain the faster time to task failure observed in the behavioral measurements of the present study.

Although the link between greater cortical output and the faster increase in EMG activity is appealing, the electrophysiological and the behavioral data cannot be directly linked because they were recorded in different experiments: for instance, we only recorded activity of the FDI and APB muscles in Experiment 1, but not from other hand and forearm muscles that could contribute to task performance. The behavior of these muscles during the time course of fatigue could be different from what we observed from the FDI and APB. Additionally, as proprioception results from the weighted sum of all available proprioceptive inputs (Boisgontier and Nougier 2013), the distinct interpretation of the augmented feedback could have modified this sensory weighting causing a differential integration of proprioceptive information, that is, interpretation-specific weighting of specific afferent sources. Finally, as position and force sense are altered in the course of muscle fatigue (Allen et al. 2007; Vuillerme and Boisgontier 2008) and also rely on different proprioceptors, the possibility for fatigue to differently affect one or the other sense cannot be ruled out.

\section{Position and force control in humans}

The question of how the central nervous system 'treats' position and force information is of ongoing debate and of great interest as it is important for many areas such as sports science, rehabilitation, and robotics. Previous studies indicated a task-specific control of force and position. For example, Milner and Hinder (2006) showed that position information, rather than force information, is used when adapting to changes in environmental dynamics (e.g., external perturbations).

Previously, Enoka and colleagues suggested that position tasks are harder to control than force tasks (for a review, see Enoka et al. 2011). However, in their paradigm, they changed not only the type of feedback (force or position) but also the compliance of the task. Thus, it could not be clarified whether the feedback or the compliance was causing these differences. Therefore, we introduced a new task where changes in position and force were proportional to each other and the compliance remained unchanged (Lauber et al. 2012). With this paradigm, we showed differences in the control of force and position tasks when the feedback resulted from a force sensor or a goniometer, respectively. In this previous study, we tried to match the information arising from the force sensor and the goniometer so that it was not possible - at least consciously_-to differentiate between the sources of information. Nevertheless, the distinct possibility existed that there might have been source-specific differences, which might have allowed subjects to unconsciously fulfill the force task better than the position task. Therefore, we could not explain in the former study why subjects treated the force and the position signal differently, or with other words: we could not clarify whether the source of feedback or other factors such as the interpretation of the signal was responsible for our findings. In the present study, we went one step further by presenting one and the same feedback within each group ( $\mathrm{pF}$ - and $\mathrm{fF}$-groups) so that we could be sure that the content of the signal was absolutely identical within these groups. As the results are similar to our previous findings, it seems that the reason for the differential organization of force- and position-controlled contractions with the current setup is in all likelihood not the source (goniometer versus force transducer) but the interpretation of the task. This assumption is further strengthened by the results of the control group, which displayed no feedback-specific differences in behavior and M1 activity despite receiving feedback from different sources.

In summary, the present study highlights the important issue that external feedback used to control movements is differently integrated dependent on the expectancy of the central nervous system, parameter of which (force or position) has to be controlled. This implies not only that it is possible to switch from a 'force control mode' to a 'position 
control mode' and vice versa, but also the possibility to alter task performance by giving specific instructions. Further studies have to test how this knowledge can be transferred to practical settings such as rehabilitation, sports, and learning of practical skills (for instance surgery).

\section{References}

Allen TJ, Ansems GE, Proske U (2007) Effects of muscle conditioning on position sense at the human forearm during loading or fatigue of elbow flexors and the role of the sense of effort. J Physiol 580:423-434

Boisgontier MB, Nougier V (2013) Proprioception: bilateral inputs first. Neurosci Let 534:96-100

Davey NJ, Romaiguere P, Maskill DW, Ellaway PH (1994) Suppression of voluntary motor activity revealed using transcranial magnetic stimulation of the motor cortex in man. J Physiol 477(2): 223-235

Di Lazzaro V, Oliviero DRA, Ferrara PPL, Mazzone AIP, Rothwell PTJC (1998) Magnetic transcranial stimulation at intensities below active motor threshold activates intracortical inhibitory circuits. Exp Brain Res 119(2):265-268

Enoka RM, Baudry S, Rudroff T, Farina D, Klass M, Duchateau J (2011) Unraveling the neurophysiology of muscle fatigue. J Electromyogr Kinesiol 21:208-219

Hopper D (2003) The influence of visual feedback on power during leg press on elite women field hockey players. Physical Therapy in Sport 4:182-186

Klass M, Lévénez M, Enoka RM, Duchateau J, Le M (2008) Spinal mechanisms contribute to differences in the time to failure of submaximal fatiguing contractions performed with different loads. J Neurophysiol 99(3):1096-1104

Langhorne P, Coupar F, Pollock A (2009) Motor recovery after stroke: a systematic review. Lancet Neurol 8:741-754

Lauber B, Leukel C, Gollhofer A, Taube W (2012) Time to task failure and motor cortical activity depend on the type of feedback in visuomotor tasks. PLoS ONE 7:e32433
Löscher WN, Nordlund MM (2002) Central fatigue and motor cortical excitability during repeated shortening and lengthening actions. Muscle Nerve 25:864-872

Milner TE, Hinder MR (2006) Position information but not force information is used in adapting to changes in environmental dynamics. J Neurophysiol 96:526-534

Moran K, Murphy C, Marshall B (2011) The need and benefit of augmented feedback on service speed in tennis. Med Sci Sports Exerc 44(4):754-760

Oldfield RC (1971) The assessment and analysis of handedness: the Edinburgh inventory. Neuropsychologia 9:97-113

Peacock B, Westers T, Walsh S, Nicholson K (1981) Feedback and maximum voluntary contraction. Ergonomics 24:223-228

Petersen NC, Butler J, Marchand-pauvert V, Fisher R, Ledebt A, Pyndt HS, Hansen NL, Nielsen JB (2001) Suppression of EMG activity by transcranial magnetic stimulation in human subjects during walking. J Physiol 537(2):651-656

Petersen NC, Butler J, Taylor JL, Gandevia SC (2010) Probing the corticospinal link between the motor cortex and motoneurones: some neglected aspects of human motor cortical function. Acta Physiol 198:403-416

Porter R, Lemon RN (1993) Corticospinal function and voluntary movement. Oxford University Press, Oxford

Rudroff T, Justice JN, Matthews S, Zuo R, Enoka RM (2010) Muscle activity differs with load compliance during fatiguing contractions with the knee extensor muscles. Exp Brain Res 203:307-316

Schmidt RA, Lee T (2011) Augmented Feedback. In: Schmidt RA, Lee T (eds) Motor control and learning-a behavioral emphasis. Human Kinetics, Champaign, pp 393-427

Scott SH (2003) The role of primary motor cortex in goal-directed movements: insights from neurophysiological studies on nonhuman primates. Curr Opin Neurobiol 13:671-677

Seifert T, Petersen NC (2010) Changes in presumed motor cortical activity during fatiguing muscle contraction in humans. Acta Physiol 199:317-325

Vuillerme N, Boisgontier M (2008) Muscle fatigue degrades force sense at the ankle joint. Gait \& posture 28:521-524

Zuur AT, Lundbye-jensen J, Leukel C, Taube W, Grey MJ, Gollhofer A, Nielsen JB, Gruber M (2010) Contribution of afferent feedback and descending drive to human hopping. J Physiol 5:799-807 